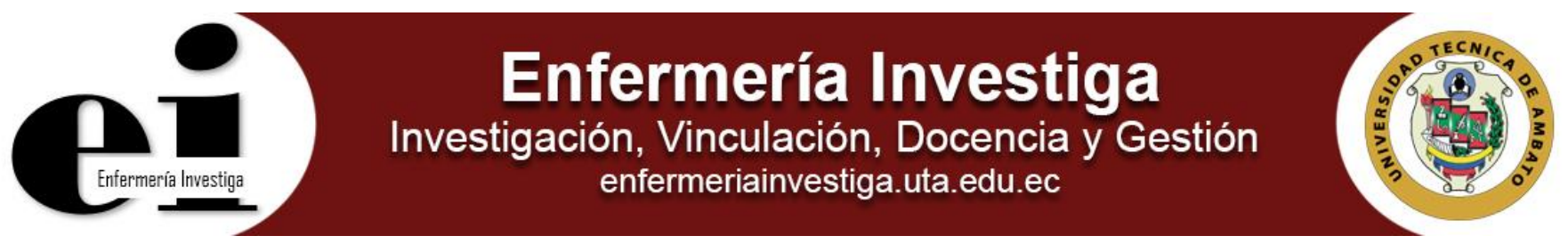

DOI: http://dx.doi.org/10.29033/enfi.v4i1.476

Artículo original

\title{
Conocimientos y prácticas sexuales asociados al VIH/SIDA en la comunidad Shuar de Uyuntza- Morona Santiago
}

Knowledges and sexual practices associated with HIV/AIDS in Shuar community of Uyuntza- Morona Santiago

\author{
Sandra Paola Nagua Uyaguari ${ }^{1}$, Doriz Angélica Jiménez Brito², Fabiola Beatriz Chasillacta Amores ${ }^{3}$ \\ ${ }^{1}$ Centro de Salud INNFA Sucua - Sucua - Morona Santiago - Ecuador \\ ${ }^{2}$ Universidad de Cuenca - Facultad de Ciencias Médicas - Carrera de Enfermería - Cuenca - Ecuador \\ ${ }^{3}$ Universidad Técnica de Ambato - Facultad de Ciencias de la Salud - Carrera de Enfermería - Ambato - Ecuador
}

Nagua SP, Jiménez DA, Chasillacta FB. Conocimientos y prácticas sexuales asociados al VIH/SIDA en la comunidad Shuar de Uyuntza- Morona Santiago. Enferm Inv. 2019; 4(1) 8-13

2477-9172 / 2550-6692 Derechos Reservados @ 2019 Universidad Técnica de Ambato, Carrera de Enfermería. Este es un artículo de acceso abierto distribuido bajo los términos de la Licencia Creative Commons, que permite uso ilimitado, distribución y reproducción en cualquier medio, siempre que la obra original es debidamente citada.

\section{Historia:}

Recibido: 22 noviembre 2018 Revisado: 23 diciembre 2018 Aceptado: 18 marzo 2019

Palabras Claves: VIH, prácticas Sexuales, conocimientos, cultura investigación cualitativa

Keywords: HIV, sexual practices, knowledges, culture, cualitive investigation

\section{Resumen}

Introducción: en Uyuntza, una comunidad indígena shuar de Morona Santiago, se constata un alto riesgo de adquirir enfermedades de transmisión sexual y VIH/SIDA debido al escaso conocimiento sobre este tema.

Objetivo: caracterizar los conocimientos y prácticas sexuales vinculadas con el VIH/SIDA, en la Comunidad Uyuntza- Morona Santiago 2018.

Métodos: grupo focal, se aplicó la metodología cualitativa, la muestra fue seleccionada por conveniencia: tres hombres y tres mujeres entre los 22 y 42 años, con vida sexual aoctiva y no diagnosticada de VIH/SIDA.

Resultados: la principal fuente de información son las charlas en los sub centros de salud. El conocimiento es limitado, existe confusión entre los conceptos VIH y SIDA. Las mujeres se limitan a expresar sus conocimientos y opiniones. Las prácticas sexuales de riesgo son frecuentes en el grupo masculino, entre estas está: el inicio precoz de las relaciones sexuales, frecuentar burdeles, la promiscuidad, infidelidad y el escaso uso de preservativo.

Conclusión: tanto hombres como mujeres de este estudio, tienen escaso conocimiento sobre el VIH/SIDA lo que los deja como una comunidad con alto riesgo de contagio y tienen la predisposición grupal por instruirse en el tema para evitar "esta terrible enfermedad".

\section{Abstract \\ Introduction: in Uyuntza, a Shuar indigenous} community of Morona Santiago, there is a high risk of acquiring sexually transmitted diseases and HIV / AIDS due to the scarce knowledge on this subject. Objective: to characterize the sexual knowledge and practices related to HIV / AIDS, in the UyuntzaMorona Santiago 2018 Community.

Methods: a focus group, the qualitative methodology was applied, the sample was selected for convenience: three men and three women between 22 and 42 years, with active sexual life and not diagnosed with HIV / AIDS.

Results: the main source of information is the talks in the health sub-centers. Knowledge is limited, there is confusion between the concepts HIV and AIDS. Women limit themselves to expressing their knowledge and opinions. The risky sexual practices are frequent in the male group, among these are: the early onset of sexual relations, frequenting brothels, promiscuity, infidelity and the scarce use of condoms.

Conclusion: both men and women in this study, have little knowledge about HIV / AIDS which leaves them as a community with high risk of infection and have the group predisposition to be educated on the subject to avoid "this terrible disease."

Fabiola Beatriz Chasillacta Amores. Universidad Técnica de Ambato, Facultad de Ciencias de la Salud, Carrera de Enfermería. Teléfono: +593 98732830, Ambato, Ecuador. Email: fb.chasillacta@uta.edu.ec 


\section{Introducción}

Partiendo de los conceptos generales se dice que el VIH (Virus de Inmunodeficiencia Humana) es el desencadenante del SIDA (Síndrome de Inmunodeficiencia Adquirida) y el SIDA es la fase más avanzada de la infección por el VIH.1 La patogénesis de la infección por el VIH comienza con la pérdida de funcionalidad de los linfocitos CD4+ con la subsecuente pérdida de la competencia del sistema inmune. ${ }^{1}$

Desde el año 1996 comenzó a llamar la atención a la mayoría de la población sobre la aparición de casos de VIH en pueblos indígenas de América. ${ }^{2}$

Según la Organización Mundial de la Salud (OMS) reportó que hubo 36.7 millones de personas infectadas por el VIH en todo el mundo, de las cuales 2.1 millones de personas contrajeron el VIH en 2015. ${ }^{3}$

En el año 2016 la Organización de las Naciones Unidas para el SIDA (ONUSIDA) informó que existen alrededor de 36.9 millones de personas que viven con $\mathrm{VIH}$ y alrededor de 2.1 millones de personas contrajeron la infección en $2014 .^{4}$

En América Latina y el Caribe en el 2014 hubo aproximadamente unas 100.000 nuevas infecciones por VIH. 5 En Venezuela en el 2011 el Instituto Venezolano de Investigaciones Científicas y el Instituto de Biomédica de la Universidad Central de Venezuela, realizaron investigaciones en comunidades de Warao Delta del Orinoco, encontrando la presencia del virus en $9.5 \%$ de los habitantes investigados, afectando principalmente a jóvenes de entre 15 y 24 años. ${ }^{5}$ En Colombia entre 1983 y 2013 se notificaron alrededor de 94.000 casos en la población general. ${ }^{6}$

En Perú Según los cálculos del Ministerio de Salud (Minsa), hay unas 20 mil personas que son VIH positivo y no lo saben. ${ }^{7}$

En comunidades indígenas de Honduras y Panamá, la prevalencia es seis veces más alta que en la población en general. Además, la epidemia se ha ido incrementando notablemente en los pueblos originarios de Guatemala, Honduras, Colombia, Venezuela, Chile, Panamá, México, Ecuador, Perú, Bolivia y en las diferentes fronteras brasileñas. ${ }^{8}$

Se tiene constancia de situaciones alarmantes de prevalencia y/o mortalidad por VIH en los siguientes casos: el pueblo Aymara de Chile; el pueblo Warao en Venezuela; los pueblos Kuna y Bugle en Panamá; y la nacionalidad Shuar en Ecuador. ${ }^{8}$

De acuerdo a proyecciones de ONUSIDA ${ }^{4}$ en el Ecuador habría entre 24000 a 41000 adultos y niños viviendo con VIH en 2016. La Subsecretaria Nacional de Vigilancia de la Salud Pública en el Ecuador destacó que existen 21 casos de SIDA y 17 casos de VIH confirmados solo en esta provincia. Según el reporte de las RPIS, para el año 2016 el Ecuador registra un total de 56.106 casos de VIH/sida acumulados; el mayor porcentaje lo reporta el MSP con el $88.29 \%$, seguido por el IESS con el $11.06 \%$; y con menores porcentajes el ISSFA y el ISSPOL con el $0.45 \%$ y $0.18 \%$, respectivamente. 9 Por lo que se pretende caracterizar los conocimientos y prácticas sexuales vinculadas con el VIH/SIDA en la Comunidad Uyuntza.

\section{Materiales y Métodos}

El estudio fue de tipo cualitativo fenomenológico cuya población de estudio fueron 6 miembros de la comunidad Shuar de Uyuntza-Morona Santiago. La muestra para integrar al grupo focal, fue seleccionada por conveniencia misma que fue constituida por tres hombres y tres mujeres entre los 22 y 42 años, con vida sexual activa y no diagnosticada de VIH/SIDA. Se empleó un temario abierto que permitió una indagación más profunda. La información fue grabada, transcrita en Word como documento primario y luego se procedió a la codificación, elaboración de memos mediante el software el software Atlas-Ti versión 7.5.4. utilizando como categorías principales conocimientos y prácticas sexuales se realizaron vínculos, redes semánticas: además, conceptos, proposiciones y valoraciones de los resultados. 


\section{Resultados}

Los ovarios son glándulas femeninas, que producen óvulos para la reproducción, además hormonas como estrógenos y progesterona. Los ovarios tienen tres tipos de tejidos: Células epiteliales que cubren el ovario.

Tabla 1: Conocimientos

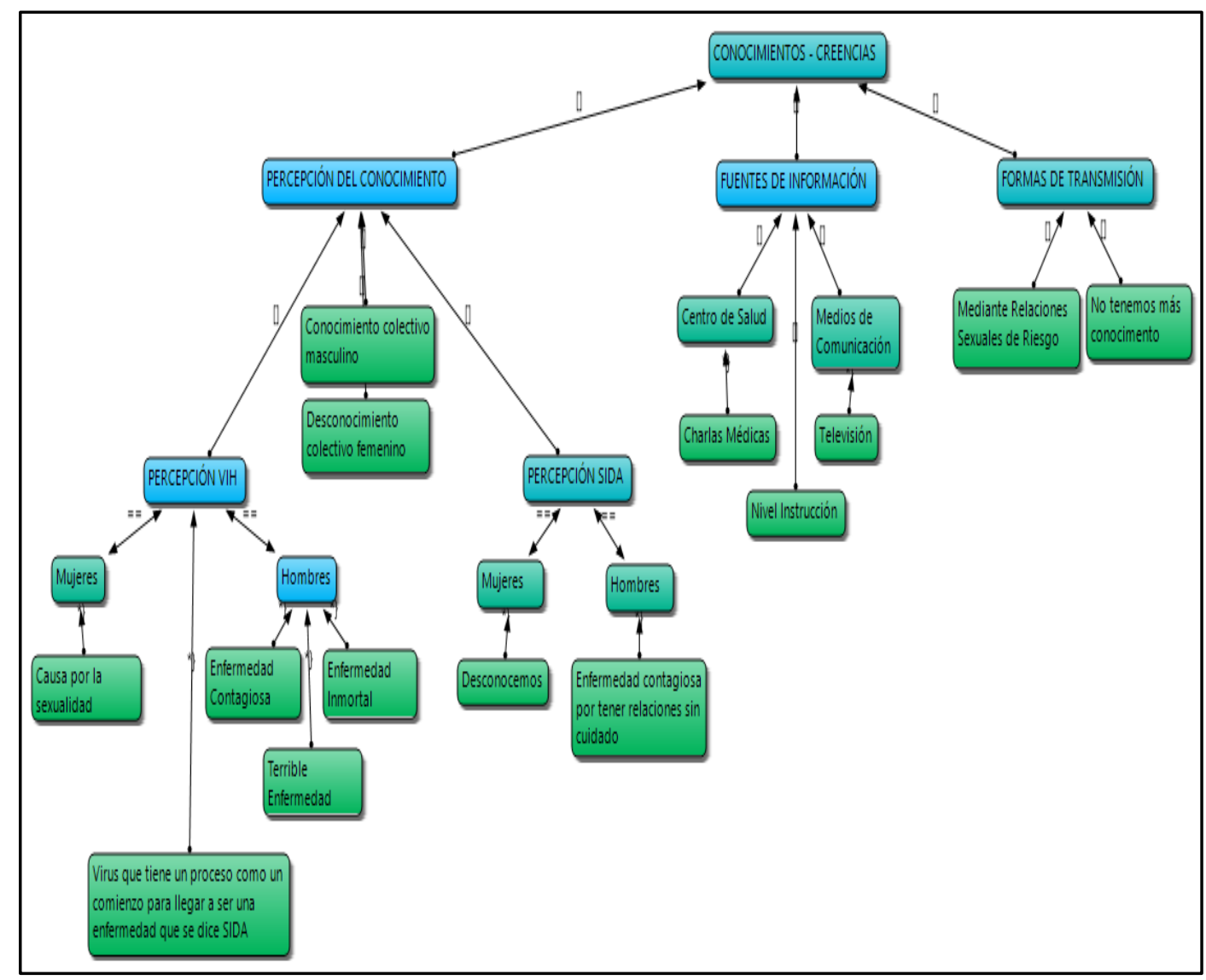

Fuente: Temario

Elaboración: Paola Nagua

De acuerdo a los participantes, la principal fuente de información sobre VIH/SIDA fueron las charlas en los subcentros de Salud, mismas que en cuestión de tiempo y calidad son insuficientes "no es cosa muy profunda". Compararon Uyuntza con comunidades cercanas, señalaron que en "otras partes" se pueden estar dando charlas por parte del personal de los centros de salud, pero "aquí no".

Ellos tenían conocimientos parciales. Los hombres se expresaron más acertadamente, participaban con más frecuencia que las mujeres; ellas decían "desconocer" o simplemente no querían responder.

Frente al tema de la definición del SIDA, la mayoría de las mujeres dijeron "no hemos escuchado nada", "desconozco", "no entendemos muy bien". Por otro lado, los hombres la identificaron como una "enfermedad contagiosa" que "se transmite por tener relaciones malas, no con cuidado".

En la comunidad existe la preocupación de llegar a contagiarse de VIH/SIDA y por la escasez de información no puedan optar por usar formas de prevención "siento que puedo tener esa enfermedad y no sé cómo prevenir" "hemos escuchado sobre esa terrible enfermedad, pero no sabemos cómo nos podemos cuidar". 
Tabla 2: Prácticas Sexuales

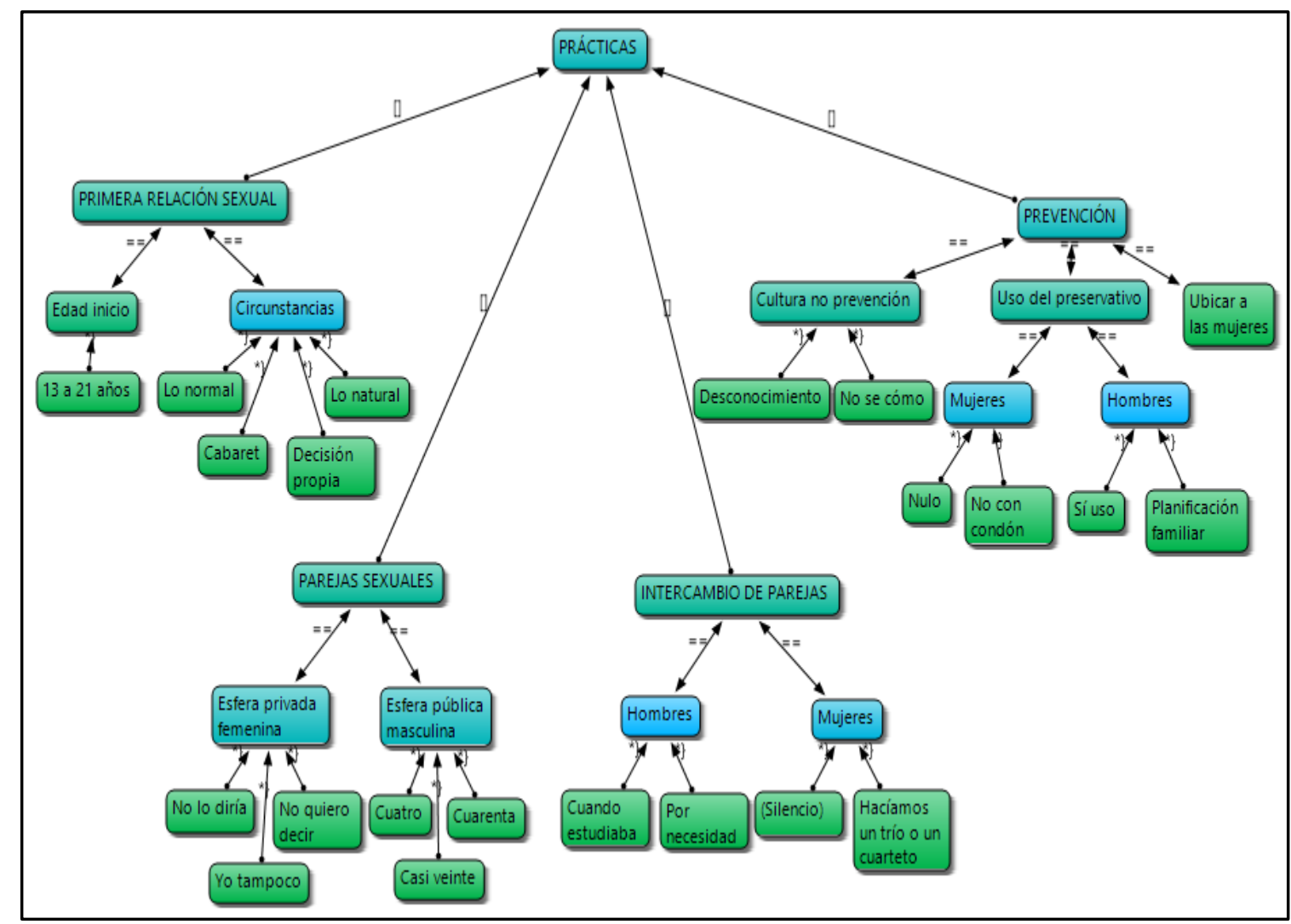

Fuente: Temario

Elaboración: Paola Nagua

La edad de inicio de las relaciones sexuales fue a los trece y la más tardía a los "21 años" en un "cabaret". En ambos casos se evidencia prácticas sexuales de riesgo, por un lado, el inicio precoz de las relaciones sexuales y por el otro frecuentar burdeles, estas acciones los dejan vulnerables a posibles contagios de VIH/SIDA y enfermedades de transmisión sexual.

Los hombres que formaron parte del estudio revelan que tienen más parejas sexuales, en uno de los casos "más de cuarenta". Las mujeres evitan expresar sus experiencias y evitan responder. Solamente una participante manifestó: "cuando era joven hacíamos un trio o un cuarteto" sin dar mayores detalles mientras que, los hombres opinan que el intercambio de parejas sexuales es frecuente y la mayoría de ellos manifestaron haber realizado esta práctica cuando eran jóvenes, bajo el contexto de los "estudios" y por "necesidad".

En los participantes existe la idea de elegir o "escoger" a sus parejas sexuales como una forma de prevención, aunque no está generalizada en el grupo. Cuando se indago acerca de métodos preventivos un participante masculino manifestó "Hay que ubicarles a las mujeres, digamos no cogiendo diferentes mujeres, por aquí o por allá",

Las mujeres de la investigación no usan preservativos ni exigen que los hombres lo hagan, prefieren lo natural "así normal ya, no con condón", algunas manifiestan sentirse vulnerables por las infidelidades de sus parejas "yo no puedo saber si él me podrá causar esa enfermedad yo no puedo saber eso".

\section{DISCUSION}

\section{Conocimientos}

La información que se obtiene de las charlas en los sub centros de salud de la comunidad, según los participantes, son insuficientes en cuestión a tiempo y calidad. Perciben limitaciones para acceder a los centros primarios de salud, aseguran sentirse "abandonados" ya que son pocas las veces que una entidad médica los visita en su comunidad concordando con la investigación sobre la epidemia de VIH en pueblos originarios de México 2016 de Muñoz Rubén y colaboradores, exalta que las instituciones del sector salud no están dando las respuestas necesarias a la problemática, las organizaciones de la sociedad civil han mostrado poco o nulo interés hacia el trabajo en las regiones indígenas y sus circuitos migratorios. ${ }^{10}$

Bolaños Gutiérrez afirma que la Vigilancia Epidemiológica de VIH/SIDA tiene acciones de educación, prevención, promoción de salud y atención médica integral. ${ }^{11}$ Sin embargo, los cumplimientos de las acciones de este programa 
se han visto disminuidos. "El fallo de la atención en el nivel primario de atención médica ha contribuido al aumento de la morbilidad por diagnósticos tardíos". ${ }^{11}$ Estas afirmaciones podrían estar relacionadas con la escasez de conocimiento e información referente a los temas de salud y VIH/SIDA en el grupo focal.

\section{Prácticas sexuales de riesgo}

Una investigación realizada por MACCHI, ML et al. Conocimientos, actitudes y prácticas acerca del VIH/SIDA del área metropolitana, Paraguay indica que las personas que inician su actividad sexual a edades muy tempranas, tienen mayor probabilidad de tener sexo con parejas con elevado riesgo para enfermedades de transmisión sexual $\mathrm{VIH} / \mathrm{SIDA}$ y menor probabilidad de utilizar condones. ${ }^{12}$

También lo afirma García Alúm en su estudio sobre Comportamiento sexual de riesgo de infección por VIH/sida en estudiantes de primer año de medicina "el individuo a esta edad, no está preparado ni física ni psicológicamente para asumir una sexualidad plena debido a la inmadurez y desconocimiento propio de estas edades por lo que tienen menos oportunidades de elegir comportamientos saludables". ${ }^{13}$ En esta investigación la edad de inicio de las relaciones sexuales fue a los trece años y otro participante señaló que su primer contacto sexual fue en un "cabaret".

En estudio como en el de Herrera C y Campero L. aborda acerca de los conocimientos frente al VIH/SIDA, y el uso del condón en grupos específicos como jóvenes o mujeres, pero en indígenas shuar específicamente se ha avanzado poco, lo que puede ser un tema para futuras investigaciones.

En el grupo focal las mujeres mantienen uniones estables con sus parejas sin embargo ellas aseguran no saber la vida sexual pasada de sus cónyuges, desconocen si ellos son fieles y por lo tanto tampoco saben si ellas podrían ser portadoras de alguna enfermedad de transmisión sexual. ${ }^{8}$ En la región de Morona Santiago en la Amazonía que cuenta con un $50 \%$ de población indígena, recientemente se notificó un incremento de los casos de VIH de un $680 \%$. De los casos registrados, el 50\% correspondía a población indígena de la nacionalidad Shuar aquí se cataloga a la mujer indígena como la principal vulnerable al contagio de enfermedades de transmisión sexual y VIH/SIDA ya que la mayoría de ellas se enfrentan a desigualdades sociales o son compañeras sexuales de personas que tienen prácticas sexuales de riesgo. "En el caso de las mujeres indígenas de Morona Santiago, la infección se produjo por vía sexual, a través de sus maridos. Ellos se infectaron pues fueron migrantes temporales para trabajar temporalmente. Se presume que ahí tenían experiencias sexuales sin protección".

\section{Conclusiones}

1. Finalmente podemos concluir que los conocimientos vinculadas con el VIH/SIDA, en un grupo focal de la Comunidad Uyuntza- Morona Santiago son limitados, mantienen prácticas sexuales de riesgo, las mujeres no usan preservativo y podrían ser compañeras sexuales de hombres que tienen múltiples parejas por lo que ellas se sienten vulnerables de ser contagiadas. Esto deja en manifiesto que el grupo es muy propenso a adquirir alguna enfermedad de transmisión sexual incluido en VIH/SIDA.

2. Es notoria la predisposición de aprender por parte de la comunidad. Existe mucha información que se podría obtener en futuras investigaciones y a través de estas planificar acciones que motive a la comunidad a crear una conciencia de prevención y así disminuir la creciente cifra de personas infectadas en la provincia de Morona Santiago.

\section{Conflicto de intereses}

Ninguno declarado por los autores.

\section{Financiación}

Autofinanciado.

\section{Agradecimientos}

Este proyecto va dirigido con una expresión de gratitud al grupo de investigadores colaboradores: la Licenciada Liliana Brito quien estuvo a cargo de la recolección y transcripción de datos, al Doctor Rosendo Rojas quien estuvo a cargo del análisis crítico del texto y a los directrices de la Facultad de Ciencias Médicas de la Universidad de Cuenca, por el apoyo constante a la Carrera de Enfermería para que se realicen proyectos como estos.

\section{Referencias}

1. Ramírez S, Enrique L. Mecanismos patogénicos de la infección por VIH. Rev Investig Clínica. abril de 2004;56(2):143-52.

2. Zambrano R, Castro D, Lozano M, Gómez N, Rojas C. Conocimientos sobre VIH y comportamientos en Salud Sexual y Reproductiva en una comunidad indígena de Antioquia. Investig Andina. 2013;15(26):640-52.

3. OMS | 10 datos sobre el VIH/sida [Internet]. WHO. [citado 18 de octubre de 2018]. Disponible en: http://www.who.int/features/factfiles/hiv/es/

4. ONUSIDA- Últimas estadísticas sobre el estado de la epidemia de sida [Internet]. [citado 5 de noviembre de 2018]. Disponible en: http://www.unaids.org/es/resources/fact-sheet

5. Mitchell C. OPS OMS | Países de América Latina y el Caribe se comprometen a reducir un $75 \%$ las nuevas infecciones por VIH en adultos y jóvenes para 2020 [Internet]. Pan American Health Organization / World Health Organization. [citado 25 de abril de 2017]. Disponible en: http://www.paho.org/hq/index.php?option=com_content\&view=article\&id=11155\%3Apaises-se-comprometen-reducir-75-nuevasinfecciones\&catid=740\%3Apress-releases\&ltemid=1926\&lang=es 
6. Castro-Arroyave DM, Gamella JF, Gómez Valencia N, Rojas Arbeláez C. CARACTERIZACION DE LA SITUACION DEL VIH/SIDA EN LOS WAYUU DE COLOMBIA: UNA APROXIMACIÓN A SUS PERCEPCIONES. Chungará Arica. marzo de 2017;49(1):109-19.

7. Juarez-Vílchez JP, Pozo EJ. Percepciones sobre comportamientos sexuales de riesgo en personas que viven con VIH/SIDA y reciben tratamiento antirretroviral en Piura, Perú. Rev Peru Med Exp Salud Publica. marzo de 2010;27(1):31-7.

8. Ponce P, Muñoz R, Stival M. Pueblos indígenas, VIH y políticas públicas en Latinoamérica: una exploración en el panorama actual de la prevalencia epidemiológica, la prevención, la atención y el seguimiento oportuno. Salud Colect. septiembre de 2017;13:537-54.

9. Informe GAM Ecuador monitoreo global del SIDA [Internet]. [citado 19 de febrero de 2019]. Disponible en: http://www.unaids.org/sites/default/files/country/documents/ECU_2017_countryreport.pdf

10. Muñoz P, Stival bernal. la epidemia de VIH en pueblos originarios de Mexico [Internet]. entrediversidades. [citado 21 de octubre de 2018 ]. Disponible en: http://entrediversidades.unach.mx/index.php/entrediversidades/article/download/6/16?inline=1

11. Bolaños Gutiérrez MR, Díaz Lemus M, Segredo Pérez A. Curso para el equipo básico de salud sobre el control y prevención del VIH/sida. Educ Médica Super. junio de 2015;29(2):0-0.

12. Macchi M, Benítez Leite S, A C, Nuñez C, Ortigoza D. Conocimientos, actitudes y prácticas acerca del VIH/SIDA en jóvenes de nivel medio de Educación, del área metropolitana, Paraguay. Rev Chil Pediatría. abril de 2008;79(2):206-17.

13. Alúm G, Esther N, Vento V, Carmen A, Machado G, Jesús A, et al. Comportamiento sexual de riesgo de infección por VIH/sida en estudiantes de primer año de medicina. Rev Cienc Médicas Pinar Río. junio de 2011;15(2):170-83.

Enferm Inv. 4(1)8-13 